\title{
A note on a question of Abe
}

\author{
by
}

Douglas B u r ke (Las Vegas, NV)

\begin{abstract}
Assuming large cardinals, we show that every $\kappa$-complete filter can be generically extended to a $V$-ultrafilter with well-founded ultrapower. We then apply this to answer a question of Abe.
\end{abstract}

1. Weakly precipitous filters. A set $\mathcal{F}$ is a filter if it is closed under intersections, $\emptyset \notin \mathcal{F}$, and whenever $A \subseteq B \subseteq \bigcup \mathcal{F}$ with $A \in \mathcal{F}$, then $B \in \mathcal{F}$. In what follows $\kappa$ is always a regular cardinal $>\omega$. A filter $\mathcal{F}$ is $\kappa$-complete iff it is closed under intersections of size $<\kappa$.

Definition 1.1. Let $\mathcal{F}$ be a $\kappa$-complete filter. We say $\mathcal{F}$ is weakly precipitous if there is a partial order $\mathbb{P}$ and a $\mathbb{P}$-name $\dot{G}$ such that it is forced that $\dot{G}$ is a $V$ - $\kappa$-complete ultrafilter extending $\mathcal{F}$ with well-founded ultrapower. We say $\mathcal{F}$ is $\alpha$-weakly precipitous if there is a partial order $\mathbb{P}$ and a $\mathbb{P}$-name $\dot{G}$ such that it is forced that $\dot{G}$ is a $V$ - $\kappa$-complete ultrafilter extending $\mathcal{F}$ with $j_{\dot{G}}(\alpha)$ in the well-founded part of the ultrapower.

If $\kappa$ is strongly compact then every $\kappa$-complete filter can be extended to a $\kappa$-complete ultrafilter. If we use a generic embedding instead of a strongly compact embedding, then (large cardinals imply that) for every $\kappa$, every $\kappa$-complete filter is weakly precipitous.

Recall that $S$ is stationary if for every $f:(\bigcup S)^{<\omega} \rightarrow \bigcup S$ there is an $a \in$ $S$ that is closed under $f$. We have $\mathbb{P}_{<\delta}=\left\{S \in V_{\delta} \mid S\right.$ is stationary $\}$, ordered by $S \leq T$ iff $\bigcup S \supseteq \bigcup T$ and for all $a \in S, a \cap(\bigcup T) \in T$. This generalization of stationary and the following theorem appear in [W1] and [W2].

Theorem 1.2 (Woodin). Assume $\delta$ is a Woodin cardinal, $G \subseteq \mathbb{P}_{<\delta}$ is generic, and $j_{G}: V \rightarrow M$ is the generic embedding. Then $M^{<\delta} \subseteq M$ in $V[G]$.

Lemma 1.3. Assume $\mathcal{F}$ is a $\kappa$-complete filter and there is a Woodin cardinal $>|\cup \mathcal{F}|$. Then $\mathcal{F}$ is weakly precipitous.

2000 Mathematics Subject Classification: Primary 03E40, 03E55. 
Proof. We may assume $\bigcup \mathcal{F}$ is a cardinal $\lambda$ and $\lambda \geq \kappa$. Let $\delta>\lambda$ be a Woodin cardinal. The forcing $\mathbb{P}$ that witnesses that $\mathcal{F}$ is weakly precipitous is

$$
\mathbb{P}_{<\delta}\left\lceil\left\{a \subseteq V_{\lambda+1}|| a \mid<\kappa \& a \cap \kappa \in \kappa\right\} .\right.
$$

Let $H \subseteq \mathbb{P}$ be generic and $j: V \rightarrow M$ the generic embedding (so $M$ is well-founded). It is easy to see (using techniques from [W1]; also see [M2], Chapter 9) that $\operatorname{cp}(j)=\kappa, j^{\prime \prime} \mathcal{F} \in M$, and $j(\kappa)>\left|j^{\prime \prime} \mathcal{F}\right|$ (this last inequality holds since $\left.\mathcal{F} \subseteq V_{\lambda+1}\right)$. Since $j(\mathcal{F})$ is a $j(\kappa)$-complete filter and $j^{\prime \prime} \mathcal{F} \subseteq j(\mathcal{F})$, there is a $c \in \bigcap j^{\prime \prime} \mathcal{F}$.

Now in $V[H]$ define a $V$-ultrafilter $\mathcal{G}$ on $\lambda$ by $A \in \mathcal{G}$ iff $c \in j(A)$. Clearly, $\mathcal{G}$ is a $V$ - $\kappa$-complete ultrafilter extending $\mathcal{F}$. Since $\operatorname{Ult}(V, \mathcal{G})$ can be embedded into $M$ (by the map $k([f])=j(f)(c))$, $\operatorname{Ult}(V, \mathcal{G})$ is well-founded. Finally, standard forcing facts give a name $\dot{G}$ for $\mathcal{G}$.

We can get by with much smaller large cardinals if all we want is $\alpha$-weakly precipitous.

Lemma 1.4. Assume $\mathcal{F}$ is a $\kappa$-complete filter and there is a measurable cardinal $\delta>|\bigcup \mathcal{F}|$. Then $\mathcal{F}$ is $\delta$-weakly precipitous.

Proof. We may assume $\bigcup \mathcal{F}=\lambda$ a cardinal and $\lambda \geq \kappa$. Since $\delta>\lambda$ is measurable,

$$
S=\left\{a \subseteq V_{\delta}|a \cap \kappa \in \kappa \&| a \cap V_{\lambda+1}|<\kappa \&| a \mid=\delta\right\}
$$

is stationary ([W1]). Let $\mathbb{P}$ be all stationary subsets of $S$ ordered by inclusion, and $H \subseteq \mathbb{P}$ generic. Then we have an embedding $j: V \rightarrow(M, E)$ with $\operatorname{cp}(j)=\kappa, \delta$ in the well-founded part of $(M, E), j(\delta)=\delta, j^{\prime \prime} \mathcal{F} \in M$, and $\left|j^{\prime \prime} \mathcal{F}\right|<j(\kappa)$ (this is all standard-see [W1] or [M2]). Now we argue as above to get a $V$ - $\kappa$-complete ultrafilter $\mathcal{G}$ extending $\mathcal{F}$. Let $j_{\mathcal{G}}: V \rightarrow \operatorname{Ult}(V, \mathcal{G})$ and $k: \operatorname{Ult}(V, G) \rightarrow(M, E)$ be the canonical maps. Then $j_{\mathcal{G}}(\delta)$ is in the well-founded part of $\operatorname{Ult}(V, G)$ since $k\left(j_{\mathcal{G}}(\delta)\right)=j(\delta)=\delta$.

2. A question of Abe. It is possible that one can use large cardinals (and weakly precipitous filters) instead of precipitous filters. For example, in [M1] Magidor proves that if there is a precipitous ideal on $\omega_{1}$ and a measurable cardinal then all $\Sigma_{3}^{1}$ sets are Lebesgue measurable. If we use Theorem 1.2 instead of a precipitous ideal on $\omega_{1}$, Magidor's proof gives that all $\Sigma_{3}^{1}$ sets are Lebesgue measurable from a measurable cardinal above a Woodin cardinal. Magidor goes on to show that all $\Sigma_{4}^{1}$ sets are Lebesgue measurable from other precipitous ideals. Using Magidor's ideas from this proof and Theorem 1.2, one sees that a measurable cardinal above $n$ Woodin cardinals implies that all $\Sigma_{n+2}^{1}$ sets are Lebesgue measurable.

In this section we give another example of this in answering a question of Abe from [A]. The following definition and two theorems are due to Abe and appear in $[\mathrm{A}]$. 
Definition 2.1 (Abe). Assume $\mathcal{F}$ is a filter on $\mathcal{P}_{\kappa} \lambda$ (all filters on $\mathcal{P}_{\kappa} \lambda$ are $\kappa$-complete and fine). $\mathcal{F}$ is weakly normal iff $\forall f$ if $\left\{a \in \mathcal{P}_{\kappa} \lambda \mid f(a) \in a\right\} \in \mathcal{F}$ then $\exists \beta<\lambda$ such that $\left\{a \in \mathcal{P}_{\kappa} \lambda \mid f(a)<\beta\right\} \in \mathcal{F}$. Further, $\mathcal{F}$ is semi-weakly normal iff $\forall f$ if $\left\{a \in \mathcal{P}_{\kappa} \lambda \mid f(a) \in a\right\} \in \mathcal{F}^{+}$then $\exists \beta<\lambda$ such that $\left\{a \in \mathcal{P}_{\kappa} \lambda \mid f(a)<\beta\right\} \in \mathcal{F}^{+}$.

Theorem 2.2 (Abe). Assume $\mathcal{F}$ is a filter on $\mathcal{P}_{\kappa} \lambda$. Then $\mathcal{F}$ is weakly normal iff $\mathcal{F}$ is semi-weakly normal and there is no sequence of $\operatorname{cof}(\lambda)$ many disjoint $\mathcal{F}$-positive sets.

TheOREM 2.3 (Abe). If $\lambda$ is regular and there is a weakly normal filter on $\mathcal{P}_{\kappa} \lambda$, then $\lambda^{<\kappa}=2^{<\kappa} \cdot \lambda$.

This last result generalizes the well-known result of Solovay [S].

Also in $[\mathrm{A}]$, Abe proved a similar result when $\operatorname{cof}(\lambda) \leq \kappa$ and asked if one can compute $\lambda^{<\kappa}$ when $\kappa<\operatorname{cof}(\lambda)<\lambda$. Abe could answer this question assuming that a certain filter was precipitous - we show that $\lambda$ weak precipitousness suffices.

Theorem 2.4. Assume $\beta$ is regular and there is a filter $\mathcal{F}$ on $\mathcal{P}_{\kappa} \beta$ that has no $\beta$ sequence of disjoint sets from $\mathcal{F}^{+}$(and there is a measurable cardinal $>\beta)$. Then there is a weakly normal filter on $\mathcal{P}_{\kappa} \beta$.

REMARK. Matsubara has proved that if there is a $\beta$ saturated precipitous ideal on $\mathcal{P}_{\kappa} \beta$ then $\beta^{<\kappa}=2^{<\kappa} \cdot \beta$ ([M3], [M4]). Our result (combined with 2.3) eliminates the precipitous assumption (at the expense of a large cardinal). We also seem to have a weaker saturation hypothesis.

Proof (of Theorem 2.4). Let $\mathcal{F}$ be a filter on $\mathcal{P}_{\kappa} \beta$ with no $\beta$ sequence of disjoint sets from $\mathcal{F}^{+}$. Since there is a measurable cardinal $>\beta$ there is a partial order $\mathbb{P}$ and a $\mathbb{P}$-name $\dot{G}$ such that $\mathbb{P}$ forces $\dot{G} \supseteq \mathcal{F}$ is a $V$ - $\kappa$-complete ultrafilter on $\mathcal{P}_{\kappa} \beta$ with $j_{\dot{G}}(\beta)$ in the well-founded part of the ultrapower. Now fix $f: \mathcal{P}_{\kappa} \beta \rightarrow$ Ord and $p \in \mathbb{P}$ such that $p \Vdash "[f]=\sup \left(j_{\dot{G}}^{\prime \prime} \beta\right)$ ".

Define a filter $\mathcal{E}$ on $\mathcal{P}_{\kappa} \beta$ by $A \in \mathcal{E}$ iff $p \Vdash A \in \dot{G}$. It is easy to see that $\mathcal{E}$ is a $\kappa$-complete fine filter on $\mathcal{P}_{\kappa} \beta, \mathcal{F} \subseteq \mathcal{E}$, and there is no $\beta$ sequence of disjoint $\mathcal{E}$ positive sets. Because $p \Vdash "[f]=\sup \left(j_{\dot{G}}^{\prime \prime} \beta\right)$ ", we have

$$
\forall \gamma<\beta\left\{a \in \mathcal{P}_{\kappa} \beta \mid f(a) \geq \gamma\right\} \in \mathcal{E} .
$$

Note that $T \in \mathcal{E}^{+}$iff $\exists q \leq p$ such that $q \Vdash T \in \dot{G}$. Using this, and again the fact that $p \Vdash "[f]=\sup \left(j_{\dot{G}}^{\prime \prime} \beta\right)$ ", we have

$$
\begin{aligned}
& \forall g \text { if }\left\{a \in \mathcal{P}_{\kappa} \beta \mid g(a)<f(a)\right\} \in \mathcal{E}^{+} \text {then } \\
& \exists \gamma<\beta \text { with }\left\{a \in \mathcal{P}_{\kappa} \beta \mid g(a)<\gamma\right\} \in \mathcal{E}^{+} .
\end{aligned}
$$

Finally, define a filter $\mathcal{D}$ on $\mathcal{P}_{\kappa} \beta$ by

$$
A \in \mathcal{D} \text { iff }\left\{a \in \mathcal{P}_{\kappa} \beta \mid a \cap f(a) \in A\right\} \in \mathcal{E} .
$$


Clearly, $\mathcal{D}$ is a $\kappa$-complete filter on $\mathcal{P}_{\kappa} \beta$. Using (1) and the fact that $\mathcal{E}$ is fine, we find that $\mathcal{D}$ is fine. Note that $A \in \mathcal{D}^{+}$iff $\left\{a \in \mathcal{P}_{\kappa} \beta \mid a \cap f(a)\right.$ $\in A\} \in \mathcal{E}^{+}$. So there is no $\beta$ sequence of disjoint $\mathcal{D}$ positive sets. Using (2) we see that $\mathcal{D}$ is semi-weakly normal. Therefore $\mathcal{D}$ is a weakly normal filter on $\mathcal{P}_{\kappa} \beta$.

Corollary 2.5. Assume $\operatorname{cof}(\lambda) \geq \kappa$ and there is a filter on $\mathcal{P}_{\kappa} \lambda$ with no $\lambda$ sequence of disjoint $\mathcal{F}$-positive sets (and there is a measurable cardinal $>\lambda$ ). Then $\lambda^{<\kappa}=2^{<\kappa} \cdot \lambda$.

Proof. If $\lambda$ is regular then we use 2.4 and 2.3 .

So assume $\kappa \leq \operatorname{cof}(\lambda)<\lambda$. Let $\mathcal{F}$ be a filter on $\mathcal{P}_{\kappa} \lambda$ with no $\lambda$ sequence of disjoint, positive sets. It is easy to see that there is a $\gamma<\lambda$ and $S \in \mathcal{F}^{+}$such that $S$ cannot be split into $\gamma$ many disjoint positive sets. Replace $\mathcal{F}$ with $\mathcal{F} \mid S$ (so there is no $\gamma$ sequence of disjoint positive sets) and take $\gamma \geq \operatorname{cof}(\lambda)$.

Now given any regular $\beta$ with $\gamma \leq \beta<\lambda$, let $\mathcal{F}_{\beta}$ be the projection of $\mathcal{F}$ to $\mathcal{P}_{\kappa} \beta\left(\mathcal{F}_{\beta}=\{\{a \cap \beta \mid a \in S\} \mid S \in \mathcal{F}\}\right)$. So $\mathcal{F}_{\beta}$ is a $\kappa$-complete fine filter on $\mathcal{P}_{\kappa} \beta$ with no $\beta$ sequence of disjoint positive sets (no $\gamma$ sequence in fact). So by 2.4 and $2.3, \beta^{<\kappa}=2^{<\kappa} \cdot \beta$.

Finally, since $\operatorname{cof}(\lambda) \geq \kappa$, we have $\lambda^{<\kappa}=\bigcup_{\beta<\lambda} \beta^{<\kappa}$, and therefore $\lambda^{<\kappa}=$ $2^{<\kappa} \cdot \lambda$.

\section{References}

[A] Y. Abe, Weakly normal ideals on $\mathcal{P}_{\kappa} \lambda$ and the singular cardinal hypothesis, Fund. Math. 143 (1993), 97-106.

[J] T. J. Jech, Set Theory, Academic Press, 1978.

[M1] M. Magidor, Precipitous ideals and $\Sigma_{4}^{1}$ sets, Israel J. Math. 35 (1980), 109-134.

[M2] D. A. Martin, Borel and Projective Games, book to be published.

[M3] Y. Matsubara, private communication.

[M4] - Saturated ideals and the singular cardinal hypothesis, J. Symbolic Logic 57 (1992), 970-974.

[S] R. M. Solovay, Strongly compact cardinals and the GCH, in: Proc. Tarski Symposium, Proc. Sympos. Pure Math. 25, Amer. Math. Soc., Providence, R.I., 1974, $365-372$.

[W1] W. H. Woodin, Supercompact cardinals and $\Sigma_{1}^{2}$ sets, handwritten notes, May 1985.

[W2] - Supercompact cardinals, sets of reals, and weakly homogeneous trees, Proc. Nat. Acad. Sci. U.S.A. 85 (1988), 6587-6591.

Department of Mathematics University of Nevada

Las Vegas, NV 89154, U.S.A.

E-mail: dburke@nevada.edu 